\title{
Catalytic Asymmetric Addition of Alkylzinc and Functionalized Dialkylzinc Reagents to Ketones
}

\author{
Sang-Jin Jeon, Hongmei Li, Celina Garcia, Lynne K. LaRochelle, and Patrick J. Walsh* \\ P. Roy and Diane T. Vagelos Laboratories, \\ University of Pennsylvania, Department of Chemistry \\ 231 South $34^{\text {th }}$ Street, Philadelphia, PA 19104-6323.
}

\section{Supporting Information}

Table of Contents

General Methods

Preparation of Dialkylzinc Reagents

General Procedure for Enantioselective Addition of Dialkylzinc

Reagents to Ketones

Characterization of New Compounds (S1-S34)

Conditions for the Determination of Enantiomeric Excesses

Determination of Absolute Stereochemistry

References

Spectra page

2

2

2

3

18

21

22

S1-S68 
General Methods. All reactions were carried out under a nitrogen atmosphere with oven-dried glassware. The progress of all reactions was monitored by thin-layer chromatography. All manipulations involving dialkylzinc and titanium(IV) isopropoxide were carried out under a dinitrogen atmosphere using standard Schlenk or vacuum line techniques. All solvents were dried through alumina columns. Titanium(IV) isopropoxide and all liquid ketone substrates were distilled prior to use. Dialkylzinc compounds except dimethyl- and diethylzinc which are commercially available were prepared by literature methods. 1.0 M dialkylzinc and 1.4 M titanium(IV) isopropoxide solutions were prepared and stored in a dry box. The ${ }^{1} \mathrm{H}$ NMR and ${ }^{13} \mathrm{C}\left\{{ }^{1} \mathrm{H}\right\}$ NMR spectra were obtained at 500 and $125 \mathrm{MHz}$, respectively. Silica gel (230-400 mesh) was used for air-flashed chromatography. Analysis of enantiomeric excess was performed using chiral GC and HPLC.

\section{Preparation of Dialkylzinc Reagents.}

Dialkylzinc compounds except dimethyl- and diethylzinc were prepared by literature method. ${ }^{1,2}$

\section{Enantioselective Addition of Dialkylzinc Reagents to Ketones.}

General Procedure. The bis(sulfonamide) ligand (10 mol\%) was weighed into the reaction vessel, and the dialkylzinc (1.0 M toluene solution, 3 equiv) and the titanium(IV) isopropoxide (1.4 M hexane solution, 1.2 equiv) were added at room temperature. After 5 min, the substrate ketone (1.0 equiv) was added neat. The homogeneous reaction mixture was stirred at room temperature. After completion, it was quenched with small amount of 
water (1-1.5 mL), dried over $\mathrm{MgSO}_{4}$, concentrated under reduced pressure, and purified by column chromatography on silica gel.

2-(tert-Butyl-dimethyl-silanyloxymethyl)-1-methyl-cyclohex-2-enol (S1).

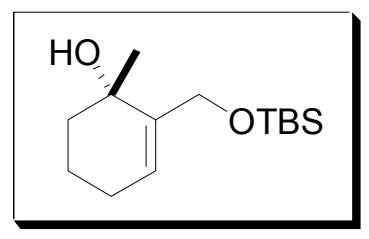

The crude product was purified by column chromatography on silica gel (hexanes / EtOAc : 95 / 5) to give the product as an oil: $[\alpha]^{20}{ }_{\mathrm{D}}=+54.3\left(c\right.$ 1.27, $\left.\mathrm{CHCl}_{3}\right) ;{ }^{1} \mathrm{H}$ NMR $\left(\mathrm{CDCl}_{3}, 500 \mathrm{MHz}\right)$

$\delta 0.07(\mathrm{~s}, 3 \mathrm{H}), 0.10(\mathrm{~s}, 3 \mathrm{H}), 0.91(\mathrm{~s}, 9 \mathrm{H}), 1.36(\mathrm{~s}, 3 \mathrm{H}), 1.52-1.61(\mathrm{~m}, 2 \mathrm{H}), 1.73-1.80(\mathrm{~m}$, 2H), 1.91-1.97 (m, 1H), 2.06-2.13 (m, 1H), 3.38 (br, 1H), 4.04 (d, $J=11.7 \mathrm{~Hz}, 1 \mathrm{H}), 4.50$ $(\mathrm{m}, 1 \mathrm{H}), 5.67(\mathrm{t}, J=3.6 \mathrm{~Hz}, 1 \mathrm{H}) \mathrm{ppm} ;{ }^{13} \mathrm{C}\left\{{ }^{1} \mathrm{H}\right\} \mathrm{NMR}\left(\mathrm{CDCl}_{3}, 125 \mathrm{MHz}\right) \delta-5.1,-5.0$, $18.6,19.6,26.0,26.3,28.4,39.0,66.6,70.0,127.0,140.0 \mathrm{ppm}$; IR (neat) 3452, 2950, 2739, 1664, 1471, 1388, 1256, $1140 \mathrm{~cm}^{-1}$; HRMS calcd for $\mathrm{C}_{14} \mathrm{H}_{27} \mathrm{OSi}(\mathrm{M}-\mathrm{OH})^{+}$: 239.1831, found 239.1838.

2-(tert-Butyl-dimethyl-silanyloxymethyl)-1-ethyl-cyclohex-2-enol (S2).

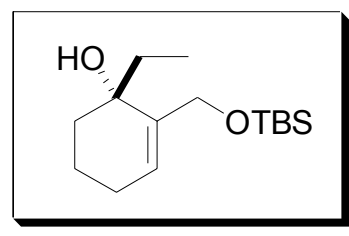

The crude product was purified by column chromatography on silica gel (hexanes / EtOAc : 95 / 5) to give the product as an oil: $[\alpha]^{20}{ }_{\mathrm{D}}=+46.0\left(c\right.$ 0.69, $\left.\mathrm{CHCl}_{3}\right) ;{ }^{1} \mathrm{H} \mathrm{NMR}\left(\mathrm{CDCl}_{3}, 500 \mathrm{MHz}\right)$

$\delta 0.08(\mathrm{~s}, 3 \mathrm{H}), 0.10(\mathrm{~s}, 3 \mathrm{H}), 0.86(\mathrm{t}, J=7.4 \mathrm{~Hz}, 3 \mathrm{H}), 0.90(\mathrm{~s}, 9 \mathrm{H}), 1.56-1.79(\mathrm{~m}, 6 \mathrm{H})$, 1.87-1.96 (m, 1H), 2.06-2.13 (m, 1H), $3.34(\mathrm{br}, 1 \mathrm{H}), 4.00(\mathrm{~d}, J=11.6 \mathrm{~Hz}, 1 \mathrm{H}), 4.45(\mathrm{~m}$, 1H), $5.76(\mathrm{~m}, 1 \mathrm{H}) \mathrm{ppm} ;{ }^{13} \mathrm{C}\left\{{ }^{1} \mathrm{H}\right\} \mathrm{NMR}\left(\mathrm{CDCl}_{3}, 125 \mathrm{MHz}\right) \delta-5.2,-5.0,8.8,18.5,19.1$, 26.0, 26.3, 33.0, 34.2, 66.9, 72.5, 128.9, 139.2 ppm; IR (neat) 3528, 2931, 2710, 1663, 1471, 1405, 1362, 1256, $1168 \mathrm{~cm}^{-1}$; HRMS calcd for $\mathrm{C}_{15} \mathrm{H}_{28} \mathrm{OSi}\left(\mathrm{M}-\mathrm{H}_{2} \mathrm{O}\right)^{+}: 252.1909$, found 252.1909 . 


\section{4-Methyl-4,5,6,7-tetrahydro-benzofuran-4-ol (S3).}

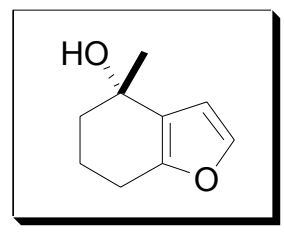

The crude product was purified by column chromatography on silica gel (hexanes / EtOAc : $90 / 10)$ to give the product as an oil: $[\alpha]^{20}=$ $+34.4\left(c\right.$ 1.33, $\left.\mathrm{CHCl}_{3}\right) ;{ }^{1} \mathrm{H} \mathrm{NMR}\left(\mathrm{C}_{6} \mathrm{D}_{6}, 500 \mathrm{MHz}\right) \delta 1.19(\mathrm{br}, 1 \mathrm{H})$, $1.36(\mathrm{~s}, 3 \mathrm{H}), 1.37-1.42(\mathrm{~m}, 2 \mathrm{H}), 1.57-1.67(\mathrm{~m}, 2 \mathrm{H}), 2.15-2.26(\mathrm{~m}, 1 \mathrm{H}), 2.30-2.39(\mathrm{~m}, 1 \mathrm{H})$, $6.24(\mathrm{~d}, J=1.7 \mathrm{~Hz}, 1 \mathrm{H}), 7.03(\mathrm{~d}, J=1.7 \mathrm{~Hz}, 1 \mathrm{H}) \mathrm{ppm} ;{ }^{13} \mathrm{C}\left\{{ }^{1} \mathrm{H}\right\} \mathrm{NMR}\left(\mathrm{C}_{6} \mathrm{D}_{6}, 125 \mathrm{MHz}\right)$ $\delta 19.9,23.3,29.1,39.3,67.5,107.9,124.5,140.9,151.5$ ppm; IR (neat) 3379, 2939, 2848, 1627, 1507, 1416, 1371, $1191 \mathrm{~cm}^{-1}$; HRMS calcd for $\mathrm{C}_{9} \mathrm{H}_{12} \mathrm{O}_{2} \mathrm{M}^{+}$: 152.0837, found 152.0838 .

\section{4-Ethyl-4,5,6,7-tetrahydro-benzofuran-4-ol (S4).}

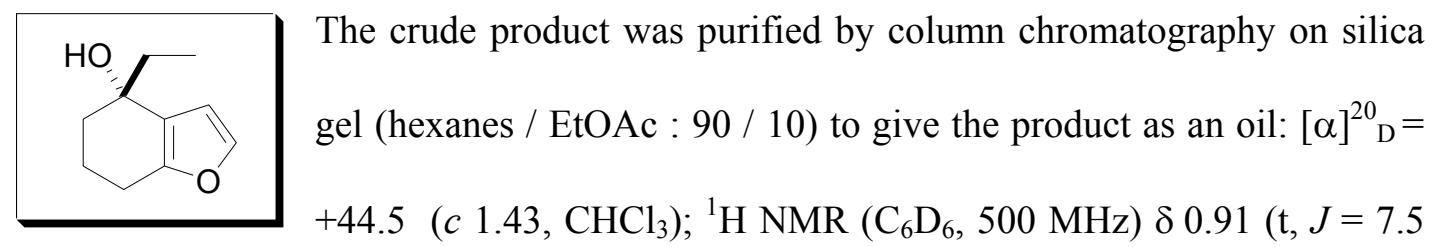

$\mathrm{Hz}, 3 \mathrm{H}), 1.15$ (br, 1H), 1.40-1.67 (m, 5H), 1.74-1.81 (m, 1H), 2.23 (ddd, $J=15.4,9.2$, $5.8 \mathrm{~Hz}, 1 \mathrm{H}), 2.37(\mathrm{dt}, J=16.2,5.0 \mathrm{~Hz}, 1 \mathrm{H}), 6.22(\mathrm{~d}, J=1.9 \mathrm{~Hz}, 1 \mathrm{H}), 7.04(\mathrm{~d}, J=1.9 \mathrm{~Hz}$, 1H) ppm; ${ }^{13} \mathrm{C}\left\{{ }^{1} \mathrm{H}\right\}$ NMR $\left(\mathrm{C}_{6} \mathrm{D}_{6}, 125 \mathrm{MHz}\right) \delta 8.7,19.7,23.4,34.5,35.7,70.1,108.2$, 123.6, 140.9, 152.2 ppm; IR (neat) 3418, 2934, 2853, 1667, 1626, 1508, 1462, $1226 \mathrm{~cm}^{-1}$; HRMS calcd for $\mathrm{C}_{10} \mathrm{H}_{13} \mathrm{O}(\mathrm{M}-\mathrm{OH})^{+}:$149.0966, found 149.0973.

\section{5-Methyl-8,9-dihydro-5H-benzocyclohepten-5-ol (S5).}

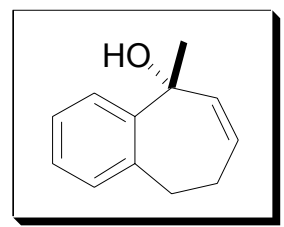

The crude product was purified by column chromatography on silica gel (hexanes / EtOAC : $95 / 5)$ to give the product as an oil: $[\alpha]^{20}=$ $+118.3\left(\right.$ c 1.10, $\left.\mathrm{CHCl}_{3}\right) ;{ }^{1} \mathrm{H} \mathrm{NMR}\left(\mathrm{C}_{6} \mathrm{D}_{6}, 500 \mathrm{MHz}\right) \delta 1.49(\mathrm{br}, 1 \mathrm{H})$, 
$1.56(\mathrm{~s}, 3 \mathrm{H}), 1.98-2.04(\mathrm{~m}, 2 \mathrm{H}), 2.62(\mathrm{dt}, J=14.0,4.2 \mathrm{~Hz}, 1 \mathrm{H}), 3.02(\mathrm{ddd}, J=14.2,9.4$, $5.7 \mathrm{~Hz}, 1 \mathrm{H})$, 5.36-5.43 (m, 1H), 5.57-5.64 (m, 1H), 6.93-6.96 (m, 1H), 7.07 (td, $J=7.4$, $1.4 \mathrm{~Hz}, 1 \mathrm{H}), 7.13-7.20(\mathrm{~m}, 1 \mathrm{H}), 7.76(\mathrm{dd}, J=7.8,1.3 \mathrm{~Hz}, 1 \mathrm{H}) \mathrm{ppm} ;{ }^{13} \mathrm{C}\left\{{ }^{1} \mathrm{H}\right\} \mathrm{NMR}\left(\mathrm{C}_{6} \mathrm{D}_{6}\right.$, $125 \mathrm{MHz}) \delta 29.1,32.0,34.6,74.5,125.3,126.6,127.4,128.8,129.9,137.6,139.3$, 147.7 ppm; IR (neat) 3375, 3014, 2931, 1655, 1484, 1455, 1367, 1234, $1114 \mathrm{~cm}^{-1}$; HRMS calcd for $\mathrm{C}_{12} \mathrm{H}_{12}\left(\mathrm{M}-\mathrm{H}_{2} \mathrm{O}\right)^{+}:$: 156.0939, found 156.0932.

\section{5-Ethyl-8,9-dihydro-5H-benzocyclohepten-5-ol (S6).}

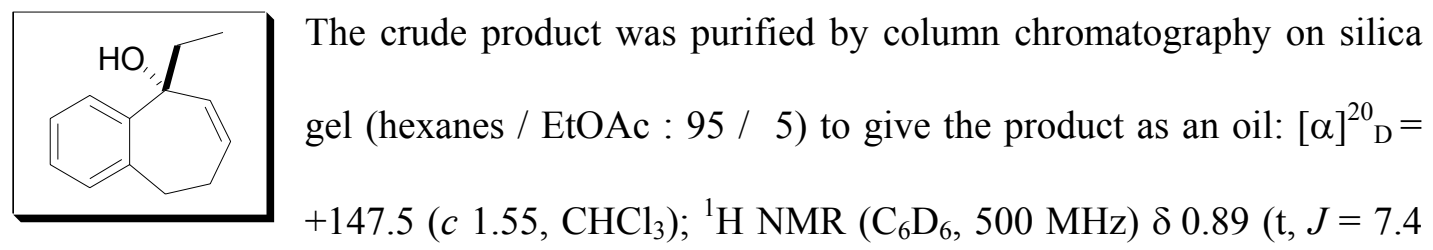

$\mathrm{Hz}, 3 \mathrm{H}), 1.34$ (br, 1H), 1.89-1.95 (m, 2H), 1.96-2.02 (m, 2H), 2.36 (dt, $J=13.8,3.8 \mathrm{~Hz}$, $1 \mathrm{H}), 3.09-3.15(\mathrm{~m}, 1 \mathrm{H}), 5.43(\mathrm{dt}, J=12.1,4.3 \mathrm{~Hz}, 1 \mathrm{H}), 5.55(\mathrm{dt}, J=12.3,1.9 \mathrm{~Hz}, 1 \mathrm{H})$, $6.94(\mathrm{~d}, J=7.3 \mathrm{~Hz}, 1 \mathrm{H}), 7.08(\mathrm{t}, J=7.3 \mathrm{~Hz}, 1 \mathrm{H}), 7.16(\mathrm{t}, J=6.8 \mathrm{~Hz}, 1 \mathrm{H}), 7.78(\mathrm{~d}, J=7.8$ $\mathrm{Hz}, 1 \mathrm{H}) \mathrm{ppm} ;{ }^{13} \mathrm{C}\left\{{ }^{1} \mathrm{H}\right\} \mathrm{NMR}\left(\mathrm{C}_{6} \mathrm{D}_{6}, 125 \mathrm{MHz}\right) \delta 8.6,29.2,34.9,36.8,77.3,126.5,126.7$, 127.4, 128.8, 129.9, 137.3, 139.1, 146.6 ppm; IR (neat) 3412, 3059, 3015, 2963, 2931, $1659,1484,1452,1373,1262 \mathrm{~cm}^{-1}$; HRMS calcd for $\mathrm{C}_{13} \mathrm{H}_{14}\left(\mathrm{M}-\mathrm{H}_{2} \mathrm{O}\right)^{+}: 170.1096$, found 170.1095.

\section{2-m-Tolyl-decan-2-ol (S7).}

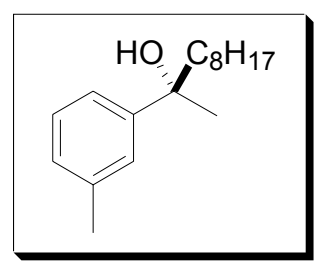

The crude product was purified by column chromatography on silica gel (hexanes / EtOAc : $90 / 10$ ) to give the product as an oil: $[\alpha]^{20}=-6.44\left(c 0.82, \mathrm{CHCl}_{3}\right) ;{ }^{1} \mathrm{H} \mathrm{NMR}\left(\mathrm{CDCl}_{3}, 500 \mathrm{MHz}\right) \delta 0.86$ $(\mathrm{t}, J=6.9 \mathrm{~Hz}, 3 \mathrm{H}), 1.08-1.33(\mathrm{~m}, 11 \mathrm{H}), 1.53(\mathrm{~s}, 3 \mathrm{H}), 1.65-1.82(\mathrm{~m}, 3 \mathrm{H}), 2.36(\mathrm{~s}, 3 \mathrm{H})$, $7.04(\mathrm{~m}, 1 \mathrm{H}), 7.18-7.26(\mathrm{~m}, 3 \mathrm{H}) \mathrm{ppm} ;{ }^{13} \mathrm{C}\left\{{ }^{1} \mathrm{H}\right\} \mathrm{NMR}\left(\mathrm{CDCl}_{3}, 125 \mathrm{MHz}\right) \delta 14.5,22.1$, 
23.0, 24.4, 29.7, 29.9, 30.4, 30.6, 32.3, 44.6, 75.1, 122.2, 125.9, 127.6, 128.4, 138.0, 148.5 ppm; IR (neat) 3408, 3023, 2919, 2854, 1607, 1487, 1455, 1373, $1147 \mathrm{~cm}^{-1}$; HRMS calcd for $\mathrm{C}_{17} \mathrm{H}_{28} \mathrm{O} \mathrm{M}^{+}$: 248.2140, found 248.2134.

6-Chloro-2-m-tolyl-hexan-2-ol (S8).

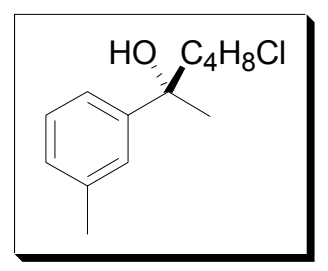

The crude product was purified by column chromatography on silica gel (hexanes / EtOAc : 90 / 10) to give the product as an oil: $[\alpha]^{20}{ }_{\mathrm{D}}=-12.9\left(c 0.77, \mathrm{CHCl}_{3}\right) ;{ }^{1} \mathrm{H} \mathrm{NMR}\left(\mathrm{CDCl}_{3}, 500 \mathrm{MHz}\right) \delta 1.21-$ $1.33(\mathrm{~m}, 1 \mathrm{H}), 1.36-1.49(\mathrm{~m}, 1 \mathrm{H}), 1.55(\mathrm{~s}, 3 \mathrm{H}), 1.69-1.83(\mathrm{~m}, 5 \mathrm{H}), 2.36(\mathrm{~s}, 3 \mathrm{H}), 3.46(\mathrm{t}, J$ $=6.8 \mathrm{~Hz}, 2 \mathrm{H}), 7.04(\mathrm{~m}, 1 \mathrm{H}), 7.17-7.29(\mathrm{~m}, 3 \mathrm{H}) \mathrm{ppm} ;{ }^{13} \mathrm{C}\left\{{ }^{1} \mathrm{H}\right\} \mathrm{NMR}\left(\mathrm{CDCl}_{3}, 125 \mathrm{MHz}\right)$ $\delta 21.9,22.1,30.6,33.3,43.8,45.2,74.9,122.2,125.9,127.8,128.5,138.2,148.1$ ppm; IR (neat) 3424, 3020, 2953, 2868, 1606, 1588, 1487, 1455, 1373,1310, $1175 \mathrm{~cm}^{-1}$; HRMS calcd for $\mathrm{C}_{13} \mathrm{H}_{18} \mathrm{Cl}(\mathrm{M}-\mathrm{OH})^{+}:$209.1097, found 209.1080.

\section{2, 2-Dimethyl-propionic acid 5-hydroxy-5-m-tolyl-hexyl-ester (S9).}

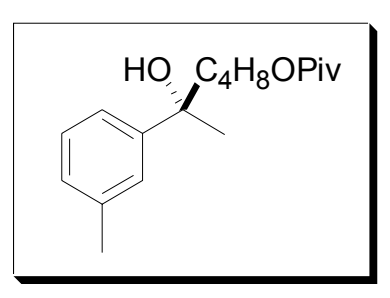

The crude product was purified by column chromatography on silica gel (hexanes / EtOAc : $90 / 10)$ to give the product as an oil: $\left[\alpha{ }^{20}{ }_{\mathrm{D}}=-6.9\left(c 1.07, \mathrm{CHCl}_{3}\right) ;{ }^{1} \mathrm{H} \mathrm{NMR}\left(\mathrm{CDCl}_{3}, 500 \mathrm{MHz}\right)\right.$ : $\delta 1.13(\mathrm{~s}, 9 \mathrm{H}), 1.25-1.29(\mathrm{~m}, 1 \mathrm{H}), 1.38(\mathrm{~s}, 3 \mathrm{H}), 1.57-1.60(\mathrm{~m}$, 1H), 1.81-1.86 (m, 4H), $2.39(\mathrm{~s}, 3 \mathrm{H}), 4.03(\mathrm{t}, J=6.5 \mathrm{~Hz}, 2 \mathrm{H}), 7.06-7.08(\mathrm{~m}, 1 \mathrm{H}), 7.23-$ $7.29(\mathrm{~m}, 3 \mathrm{H}) \mathrm{ppm} ;{ }^{13} \mathrm{C}\left\{{ }^{1} \mathrm{H}\right\} \mathrm{NMR}\left(\mathrm{CDCl}_{3}, 125 \mathrm{MHz}\right): \delta 20.8,22.0,27.6,29.3,30.6,39.1$, $44.1,64.4,74.9,122.2,125.8,127.7,128.5,138.1,148.2,179.0$ ppm; IR (neat) 3499 , 2963, 2870, $1727 \mathrm{~cm}^{-1}$; HRMS calcd for $\mathrm{C}_{18} \mathrm{H}_{28} \mathrm{O}_{3} \mathrm{Na}(\mathrm{M}+\mathrm{Na})^{+}$: 315.1936, found 315.1940 .

2-Naphthalen-2-yl-decan-2-ol (S10). 


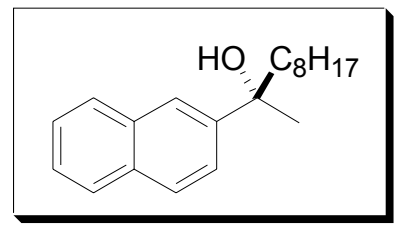

The crude product was purified by column chromatography on silica gel (hexanes / EtOAc : $90 / 10$ ) to give the product as an oil: $[\alpha]^{20}{ }_{\mathrm{D}}=-0.65\left(c 0.77, \mathrm{CHCl}_{3}\right) ;{ }^{1} \mathrm{H}$ NMR $\left(\mathrm{CDCl}_{3}, 500\right.$

MHz) $\delta 0.86(\mathrm{t}, J=6.9 \mathrm{~Hz}, 3 \mathrm{H}), 1.09-1.36(\mathrm{~m}, 11 \mathrm{H}), 1.65(\mathrm{~s}, 3 \mathrm{H}), 1.79-1.96(\mathrm{~m}, 3 \mathrm{H})$, 7.41-7.59 (m, 3H), 7.76-7.93 (m, 4H) ppm; ${ }^{13} \mathrm{C}\left\{{ }^{1} \mathrm{H}\right\}$ NMR $\left(\mathrm{CDCl}_{3}, 125 \mathrm{MHz}\right) \delta 14.5$, $23.0,24.4,29.6,29.9,30.4,30.7,32.2,44.5,75.3,123.5,124.1,126.0,126.4,127.9$, 128.2, 128.5, 132.6, 133.6, 145.9 ppm; IR (neat) 3422, 3056, 2918, 2852, 1674, 1633, 1599, 1505, 1455, $1373 \mathrm{~cm}^{-1}$; HRMS calcd for $\mathrm{C}_{20} \mathrm{H}_{28} \mathrm{O} \mathrm{M}^{+}:$284.2140, found 284.2147.

6-Chloro-2-naphthalen-2-yl-hexan-2-ol (S11).

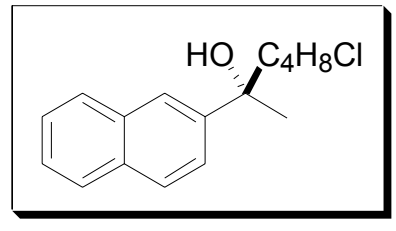

The crude product was purified by column chromatography on silica gel (hexanes / EtOAc : 90 / 10) to give the product as an oil: $[\alpha]^{20}{ }_{\mathrm{D}}=-8.82\left(c\right.$ 1.10, $\left.\mathrm{CHCl}_{3}\right) ;{ }^{1} \mathrm{H}$ NMR $\left(\mathrm{CDCl}_{3}, 500\right.$

MHz) $\delta 1.26-1.36(\mathrm{~m}, 1 \mathrm{H}), 1.43-1.52(\mathrm{~m}, 1 \mathrm{H}), 1.69(\mathrm{~s}, 3 \mathrm{H}), 1.71-1.79(\mathrm{~m}, 2 \mathrm{H}), 1.85-1.99$ $(\mathrm{m}, 3 \mathrm{H}), 3.47(\mathrm{t}, J=6.7 \mathrm{~Hz}, 2 \mathrm{H}), 7.47-7.56(\mathrm{~m}, 3 \mathrm{H}), 7.83-7.87(\mathrm{~m}, 3 \mathrm{H}), 7.93(\mathrm{~s}, 1 \mathrm{H})$ ppm; ${ }^{13} \mathrm{C}\left\{{ }^{1} \mathrm{H}\right\} \mathrm{NMR}\left(\mathrm{CDCl}_{3}, 125 \mathrm{MHz}\right) \delta 21.8,30.6,33.1,43.4,45.1,75.0,123.4,123.8$, 126.0, 126.4, 127.8, 128.3, 128.4, 132.5, 133.4, 145.3 ppm; IR (neat) 3426, 3056, 2953, 2868, 1632, 1599, 1455, $1309 \mathrm{~cm}^{-1}$; HRMS calcd for $\mathrm{C}_{16} \mathrm{H}_{19} \mathrm{ClO} \mathrm{M}^{+}$: 262.1124, found 262.1126.

2, 2-Dimethyl-propionic acid 5-hydroxy-5-(3-trifluoromethyl-phenyl)-hexyl-ester (S12).

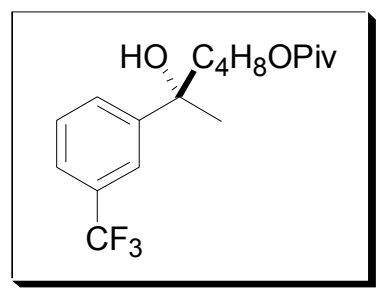

The crude product was purified by column chromatography on silica gel (hexanes / EtOAc : 90 / 10) to give the product as an 
oil: $[\alpha]^{20}{ }_{D}=-4.4\left(c 1.15, \mathrm{CHCl}_{3}\right) ;{ }^{1} \mathrm{H}$ NMR $\left(\mathrm{CDCl}_{3}, 500 \mathrm{MHz}\right): \delta 1.15(\mathrm{~s}, 9 \mathrm{H}), 1.33-1.37$ (m, 1H), 1.51-1.62 (m, 5H), 1.78-1.85 (m, 3H), $4.00(\mathrm{t}, J=6.4 \mathrm{~Hz}, 2 \mathrm{H}), 7.43-7.46(\mathrm{~m}$, $1 \mathrm{H})$, 7.49-7.50 (m, 1H), 7.58-7.60 (m, 1H), $7.71(\mathrm{~s}, 1 \mathrm{H}) \mathrm{ppm} ;{ }^{13} \mathrm{C}\left\{{ }^{1} \mathrm{H}\right\} \mathrm{NMR}\left(\mathrm{CDCl}_{3}\right.$, $125 \mathrm{MHz}): \delta$ 20.6, 27.5, 29.1, 30.7, 39.1, 44.0, 64.2, 74.8, 122.1 (d, $J=3.75 \mathrm{~Hz}), 123.8$ (d, $J=3.75 \mathrm{~Hz}), 125.7,128.7,129.0,131.0(\mathrm{q}, J=31.2 \mathrm{~Hz}), 149.2,179.0 \mathrm{ppm}$; IR (neat) 3488, 2971, 2872, 1709, $1481 \mathrm{~cm}^{-1}$; HRMS calcd for $\mathrm{C}_{18} \mathrm{H}_{24} \mathrm{~F}_{3} \mathrm{O}_{2}(\mathrm{M}-\mathrm{OH})^{+}: 329.1729$, found 329.1734 .

5-Octyl-8,9-dihydro-5H-benzocyclohepten-5-ol (S13).

The crude product was purified by column chromatography on silica
gel (hexanes / EtOAc : $95 / 5)$ to give the product as an oil: $[\alpha]^{20}{ }_{\mathrm{D}}=$
$+94.9(c 0.71, \mathrm{MeOH}) ;{ }^{1} \mathrm{H}$ NMR $\left(\mathrm{C}_{6} \mathrm{D}_{6}, 500 \mathrm{MHz}\right) \delta 0.87(\mathrm{t}, J=7.0$
$\mathrm{Hz}, 3 \mathrm{H}), 1.11-1.38$ (m, 11H), 1.45 (br, 1H), 1.51-1.62 (m, 1H), 1.93-2.08 (m, 4H), 2.40 (dt, $J=14.0,3.4 \mathrm{~Hz}, 1 \mathrm{H}), 3.22$ (ddd, $J=16.4,11.3,5.2 \mathrm{~Hz}, 1 \mathrm{H}), 5.43-5.48$ (m, $1 \mathrm{H}), 5.59-$ $5.63(\mathrm{~m}, 1 \mathrm{H}), 6.96(\mathrm{~d}, J=6.5 \mathrm{~Hz}, 1 \mathrm{H}), 7.09(\mathrm{td}, J=7.4,1.3 \mathrm{~Hz}, 1 \mathrm{H}), 7.18$ (td, $J=7.4,1.3$ $\mathrm{Hz}, 1 \mathrm{H}), 7.82(\mathrm{~d}, J=6.5 \mathrm{~Hz}, 1 \mathrm{H}) \mathrm{ppm} ;{ }^{13} \mathrm{C}\left\{{ }^{1} \mathrm{H}\right\} \mathrm{NMR}\left(\mathrm{C}_{6} \mathrm{D}_{6}, 125 \mathrm{MHz}\right) \delta 14.4,23.1$, $24.4,29.3,29.7,30.0,30.5,32.3,34.9,44.4,77.3,126.5,126.6,127.4,128.6,129.9$, 137.6, 139.1, 147.0 ppm; IR (neat) 3429, 3058, 3016, 2920, 2854, 1721, 1660, 1599, 1484, $1454 \mathrm{~cm}^{-1}$; HRMS calcd for $\mathrm{C}_{19} \mathrm{H}_{26} \mathrm{O}\left(\mathrm{M}-\mathrm{H}_{2} \mathrm{O}\right)^{+}: 254.2034$, found 254.2017.

\section{5-(4-Chloro-butyl)-8,9-dihydro-5H-benzocyclohepten-5-ol (S14).}

gel (hexanes / EtOAc : $95 / 5)$ to give the product as an oil: $[\alpha]^{20}{ }_{\mathrm{D}}=$
$+160.3\left(\mathrm{c} 0.98, \mathrm{CHCl}_{3}\right) ;{ }^{1} \mathrm{H}$ NMR $\left(\mathrm{C}_{6} \mathrm{D}_{6}, 500 \mathrm{MHz}\right) \delta 1.19-1.56(\mathrm{~m}$, $5 \mathrm{H}), 1.72-1.82(\mathrm{~m}, 2 \mathrm{H}), 1.93-2.05(\mathrm{~m}, 2 \mathrm{H}), 2.37$ (dt, $J=13.9,3.4 \mathrm{~Hz}, 1 \mathrm{H}), 3.00(\mathrm{t}, J=$ 
$6.7 \mathrm{~Hz}, 2 \mathrm{H}), 3.11(\mathrm{td}, J=13.7,4.4 \mathrm{~Hz}, 1 \mathrm{H}), 5.39-5.45(\mathrm{~m}, 1 \mathrm{H}), 5.45-5.53(\mathrm{~m}, 1 \mathrm{H}), 6.94$ $(\mathrm{d}, J=7.3 \mathrm{~Hz}, 1 \mathrm{H}), 7.05-7.11(\mathrm{~m}, 1 \mathrm{H}), 7.11-7.23(\mathrm{~m}, 1 \mathrm{H}), 7.74(\mathrm{dd}, J=7.8,1.2 \mathrm{~Hz}, 1 \mathrm{H})$ ppm; ${ }^{13} \mathrm{C}\left\{{ }^{1} \mathrm{H}\right\}$ NMR $\left(\mathrm{C}_{6} \mathrm{D}_{6}, 125 \mathrm{MHz}\right) \delta 21.7,29.1,33.1,34.8,43.2,44.7,76.9,126.4$, $126.5,127.4,128.9,129.9,137.0,138.9,146.6$ ppm; IR (neat) 3427, 3016, 2955, 1483, 1451, 1311, $1057 \mathrm{~cm}^{-1}$; HRMS calcd for $\mathrm{C}_{15} \mathrm{H}_{17} \mathrm{Cl}\left(\mathrm{M}-\mathrm{H}_{2} \mathrm{O}\right)^{+}: 232.1019$, found 232.1011.

2,2-Dimethyl-propionic acid 4-(5-hydroxy-8,9-dihydro-5H-benzocyclohepten-5-yl)-

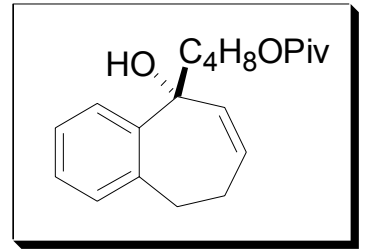

\section{butyl ester (S15).}

The crude product was purified by column chromatography on silica gel (hexanes / EtOAc : 95 / 5) to give the product as an oil: $[\alpha]^{20}{ }_{\mathrm{D}}=+81.3(c$ 0.48, MeOH$) ;{ }^{1} \mathrm{H}$ NMR $\left(\mathrm{C}_{6} \mathrm{D}_{6}, 500 \mathrm{MHz}\right) \delta 1.14(\mathrm{~s}, 9 \mathrm{H}), 1.14-1.53(\mathrm{~m}$, 5H), $1.85(\mathrm{dd}, J=9.0,7.5 \mathrm{~Hz}, 2 \mathrm{H}), 1.93-2.05(\mathrm{~m}, 2 \mathrm{H}), 2.37(\mathrm{dt}, J=14.0,3.4 \mathrm{~Hz}, 1 \mathrm{H})$, 3.09-3.21 (m, 1H), $3.92(\mathrm{t}, J=6.4 \mathrm{~Hz}, 2 \mathrm{H}), 5.39-5.44(\mathrm{~m}, 1 \mathrm{H}), 5.51-5.56(\mathrm{~m}, 1 \mathrm{H}), 6.94(\mathrm{~d}$, $J=7.3 \mathrm{~Hz}, 1 \mathrm{H}), 7.06-7.09(\mathrm{~m}, 1 \mathrm{H}), 7.13-7.20(\mathrm{~m}, 1 \mathrm{H}), 7.76(\mathrm{dd}, J=7.8,1.3 \mathrm{~Hz}, 1 \mathrm{H})$ ppm; ${ }^{13} \mathrm{C}\left\{{ }^{1} \mathrm{H}\right\} \operatorname{NMR}\left(\mathrm{C}_{6} \mathrm{D}_{6}, 125 \mathrm{MHz}\right) \delta 20.6,27.4,29.21,29.23,34.8,38.8,43.5,64.0$, 77.0, 126.51, 126.53, 127.4, 128.8, 129.9, 137.3, 139.0, 146.7, 177.7 ppm; IR (neat) 3494, $3058,3014,2957,1727,1599,1480,1455,1286 \mathrm{~cm}^{-1}$; HRMS calcd for $\mathrm{C}_{20} \mathrm{H}_{28} \mathrm{O}_{3} \mathrm{M}^{+}$: 316.2038, found 316.2034.

\section{4-Octyl-4,5,6,7-tetrahydro-benzofuran-4-ol (S16).}

The crude product was purified by column chromatography on silica gel (hexanes /

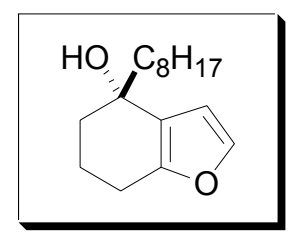
EtOAc : 95 / 5) to give the product as an oil: $[\alpha]^{20}{ }_{\mathrm{D}}=+14.7(c$ 0.97, $\left.\mathrm{CHCl}_{3}\right) ;{ }^{1} \mathrm{H}$ NMR $\left(\mathrm{C}_{6} \mathrm{D}_{6}, 500 \mathrm{MHz}\right) \delta 0.90(\mathrm{t}, J=6.7 \mathrm{~Hz}, 3 \mathrm{H}), 1.20-$ $1.44(\mathrm{~m}, 12 \mathrm{H}), 1.45-1.57(\mathrm{~m}, 3 \mathrm{H}), 1.58-1.74(\mathrm{~m}, 3 \mathrm{H}), 1.75-1.89(\mathrm{~m}$, $1 \mathrm{H}), 2.24-2.31(\mathrm{~m}, 1 \mathrm{H}), 2.32-2.47(\mathrm{~m}, 1 \mathrm{H}), 6.27(\mathrm{~d}, J=1.7 \mathrm{~Hz}, 1 \mathrm{H}), 7.05(\mathrm{~d}, J=1.7,1 \mathrm{H})$ 
ppm; ${ }^{13} \mathrm{C}\left\{{ }^{1} \mathrm{H}\right\}$ NMR $\left(\mathrm{C}_{6} \mathrm{D}_{6}, 125 \mathrm{MHz}\right) \delta 14.4,19.8,23.1,23.4,24.6,29.8,30.1,30.8$, 32.4, 36.4, 42.2, 69.9, 108.3, 123.9, 140.9, 152.0 ppm; IR (neat) 3416, 2916, 1626, 1508, $1418,1378 \mathrm{~cm}^{-1}$; HRMS calcd for $\mathrm{C}_{16} \mathrm{H}_{25} \mathrm{O}(\mathrm{M}-\mathrm{OH})^{+}: 233.1905$, found 233.1910 .

4-(4-Chloro-butyl)-4,5,6,7-tetrahydro-benzofuran-4-ol (S17).

The crude product was purified by column chromatography on silica gel (hexanes /

\begin{tabular}{c|l}
$\left.\mathrm{CHCl}_{3}\right) ;{ }^{1} \mathrm{H}$ NMR $\left(\mathrm{C}_{6} \mathrm{D}_{6}, 500 \mathrm{MHz}\right) \delta 1.21-1.65(\mathrm{~m}, 11 \mathrm{H}), 2.22(\mathrm{ddd}$, \\
$J=15.0,8.8,5.5 \mathrm{~Hz}, 1 \mathrm{H}), 2.36(\mathrm{~m}, 1 \mathrm{H}), 3.12(\mathrm{t}, J=6.4 \mathrm{~Hz}, 2 \mathrm{H}), 6.20$
\end{tabular}
$(\mathrm{d}, J=1.8 \mathrm{~Hz}, 1 \mathrm{H}), 7.04(\mathrm{~d}, J=1.8 \mathrm{~Hz}, 1 \mathrm{H}) \mathrm{ppm} ;{ }^{13} \mathrm{C}\left\{{ }^{1} \mathrm{H}\right\} \mathrm{NMR}\left(\mathrm{C}_{6} \mathrm{D}_{6}, 125 \mathrm{MHz}\right)$ $\delta$ 19.6, 21.7, 23.3, 33.3, 36.2, 41.0, 44.8, 69.6, 108.1, 123.5, 140.9, 152.0 ppm; IR (neat) $3424,2927,2851,1661,1508,1456,1260,1118 \mathrm{~cm}^{-1}$; HRMS calcd for $\mathrm{C}_{12} \mathrm{H}_{15} \mathrm{Cl}$ (M$\left.\mathrm{H}_{2} \mathrm{O}\right)^{+}: 210.0811$, found 210.0808 .

\section{2,2-Dimethyl-propionic acid 4-(4-hydroxy-4,5,6,7-tetrahydro-benzofuran-4-yl)-butyl ester (S18).}

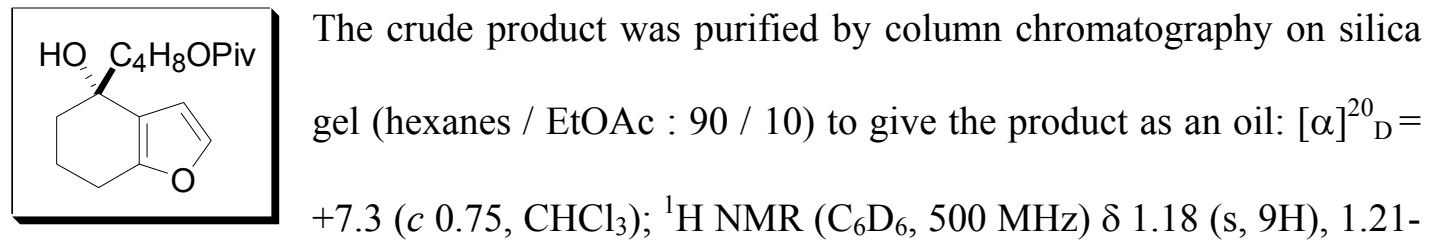

$1.39(\mathrm{~m}, 2 \mathrm{H}), 1.39-1.65(\mathrm{~m}, 8 \mathrm{H}), 1.69-1.75(\mathrm{~m}, 1 \mathrm{H}), 2.14-2.28(\mathrm{~m}, 1 \mathrm{H}), 2.31-2.42(\mathrm{~m}$, $1 \mathrm{H}), 4.02(\mathrm{t}, J=6.1 \mathrm{~Hz}, 2 \mathrm{H}), 6.22(\mathrm{~d}, J=1.8 \mathrm{~Hz}, 1 \mathrm{H}), 7.04(\mathrm{~d}, J=1.8 \mathrm{~Hz}, 1 \mathrm{H}) \mathrm{ppm}$; ${ }^{13} \mathrm{C}\left\{{ }^{1} \mathrm{H}\right\}$ NMR $\left(\mathrm{C}_{6} \mathrm{D}_{6}, 125 \mathrm{MHz}\right) \delta 19.7,20.8,23.4,27.4,29.6,36.3,38.8,41.5,64.2$, 69.8, 108.2, 123.7, 140.9, 152.0, 177.8 ppm; IR (neat) 3430, 2939, 2869, 1726, 1479, 1458, 1286, $1160 \mathrm{~cm}^{-1}$; HRMS calcd for $\mathrm{C}_{17} \mathrm{H}_{26} \mathrm{O}_{4} \mathrm{Na}(\mathrm{M}+\mathrm{Na})^{+}: 317.1729$, found 317.1741. 


\section{2,4,4-Trimethyl-1-octyl-cyclohex-2-enol (S19).}

The crude product was purified by column chromatography on silica
$+24.5\left(c 0.67, \mathrm{CHCl}_{3}\right) ;{ }^{1} \mathrm{H}$ NMR $\left(\mathrm{C}_{6} \mathrm{D}_{6}, 500 \mathrm{MHz}\right) \delta 0.90(\mathrm{t}, J=6.8 \mathrm{~Hz}$,

$3 \mathrm{H}), 0.91(\mathrm{~s}, 3 \mathrm{H}), 0.95(\mathrm{~s}, 3 \mathrm{H}), 1.19-1.45(\mathrm{~m}, 14 \mathrm{H}), 1.46-1.66(\mathrm{~m}, 4 \mathrm{H}), 1.71(\mathrm{~s}, 3 \mathrm{H}), 1.83$

(ddd, $J=13.7,11.3,3.4 \mathrm{~Hz}, 1 \mathrm{H}), 5.15(\mathrm{~s}, 1 \mathrm{H}) \mathrm{ppm} ;{ }^{13} \mathrm{C}\left\{{ }^{1} \mathrm{H}\right\} \mathrm{NMR}\left(\mathrm{C}_{6} \mathrm{D}_{6}, 125 \mathrm{MHz}\right)$

$\delta 14.4,18.1,23.1,24.5,28.4,29.8,30.1,30.6,30.8,32.27,32.33,32.8,34.1,39.5,71.9$, 136.1, 136.2 ppm; IR (neat) 3392, 2925, 2856, 1643, 1454, 1376, $1359 \mathrm{~cm}^{-1}$; HRMS calcd for $\mathrm{C}_{17} \mathrm{H}_{31}(\mathrm{M}-\mathrm{OH})^{+}$: 235.2426, found 235.2425.

\section{1-(4-Chloro-butyl)-2,4,4-trimethyl-cyclohex-2-enol (S20).}

The crude product was purified by column chromatography on silica
$+34.9\left(\mathrm{c} 1.43, \mathrm{CHCl}_{3}\right) ;{ }^{1} \mathrm{H}$ NMR $\left(\mathrm{C}_{6} \mathrm{D}_{6}, 500 \mathrm{MHz}\right) \delta 0.88(\mathrm{~s}, 3 \mathrm{H}), 0.93$
$+\mathrm{C}_{4} \mathrm{H}_{8} \mathrm{Cl}$

(s, 3H), 1.14-1.19 (m, 1H), 1.26-1.48 (m, 9H), 1.64 (s, 3H), 1.71 (ddd, $J=13.9,11.0,2.9$ $\mathrm{Hz}, 1 \mathrm{H}), 3.10(\mathrm{t}, J=6.5 \mathrm{~Hz}, 2 \mathrm{H}), 5.12(\mathrm{~s}, 1 \mathrm{H}) \mathrm{ppm} ;{ }^{13} \mathrm{C}\left\{{ }^{1} \mathrm{H}\right\} \mathrm{NMR}\left(\mathrm{C}_{6} \mathrm{D}_{6}, 125 \mathrm{MHz}\right)$ $\delta 17.9,21.6,28.4,30.5,32.2,32.6,33.4,33.9,38.3,44.8,71.6,135.9,136.3$ ppm; IR (neat) $3406,2956,1456,1309,1234,1184 \mathrm{~cm}^{-1}$; HRMS calcd for $\mathrm{C}_{13} \mathrm{H}_{22} \mathrm{Cl}(\mathrm{M}-\mathrm{OH})^{+}$: 213.1410, found 213.1401.

2,2-Dimethyl-propionic acid 4-(1-hydroxy-2,4,4-trimethyl-cyclohex-2-enyl)-butyl $\mathrm{HO}, \mathrm{C}_{4} \mathrm{H}_{8} \mathrm{OPiv}$ ester (S21). The crude product was purified by column chromatography on silica gel (hexanes / EtOAc : $90 / 10)$ to give the product as an oil: $[\alpha]^{20}{ }_{\mathrm{D}}=+24.8\left(c 0.60, \mathrm{CHCl}_{3}\right) ;{ }^{1} \mathrm{H}$ NMR $\left(\mathrm{C}_{6} \mathrm{D}_{6}\right.$, 
$500 \mathrm{MHz}) \delta 0.89(\mathrm{~s}, 3 \mathrm{H}), 0.93(\mathrm{~s}, 3 \mathrm{H}), 1.19(\mathrm{~s}, 9 \mathrm{H}), 1.26-1.55(\mathrm{~m}, 10 \mathrm{H}), 1.65(\mathrm{~s}, 3 \mathrm{H})$, $1.74(\mathrm{ddd}, J=13.9,10.8,3.1 \mathrm{~Hz}, 1 \mathrm{H}), 3.96-4.05(\mathrm{~m}, 2 \mathrm{H}), 5.13(\mathrm{~s}, 1 \mathrm{H}) \mathrm{ppm} ;{ }^{13} \mathrm{C}\left\{{ }^{1} \mathrm{H}\right\}$ NMR $\left(\mathrm{C}_{6} \mathrm{D}_{6}, 125 \mathrm{MHz}\right) \delta 17.9,20.6,26.9,27.4,28.4,29.6,30.4,32.2,32.6,33.9,38.7$, 64.1, 71.6, 135.9, 136.1, 177.7 ppm; IR (neat) 3438, 2955, 2865, 1729, 1638, 1480, 1460, 1360, 1286, $1159 \mathrm{~cm}^{-1}$; HRMS calcd for $\mathrm{C}_{18} \mathrm{H}_{32} \mathrm{O}_{3} \mathrm{Na}(\mathrm{M}+\mathrm{Na})^{+}$: 319.2249, found 319.2243 .

2-(tert-Butyl-dimethyl-silanyloxymethyl)-1-octyl-cyclohex-2-enol (S22).

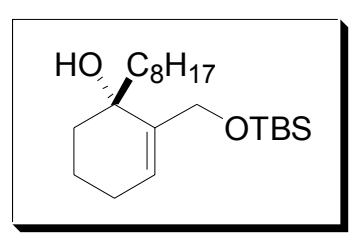

The crude product was purified by column chromatography on silica gel (hexanes / EtOAc : 95 / 5) to give the product as an oil: $[\alpha]^{20}{ }_{\mathrm{D}}=+26.2(c \quad 0.97, \mathrm{MeOH}) ;{ }^{1} \mathrm{H} \mathrm{NMR}\left(\mathrm{CDCl}_{3}, 500 \mathrm{MHz}\right)$

$\delta 0.04(\mathrm{~s}, 3 \mathrm{H}), 0.06(\mathrm{~s}, 3 \mathrm{H}), 0.91(\mathrm{t}, J=7.8 \mathrm{~Hz}, 3 \mathrm{H}), 0.93(\mathrm{~s}, 9 \mathrm{H}), 1.22-1.42(\mathrm{~m}, 10 \mathrm{H})$, $1.49-1.65(\mathrm{~m}, 3 \mathrm{H}), 1.69-1.92(\mathrm{~m}, 7 \mathrm{H}), 3.10(\mathrm{br}, 1 \mathrm{H}), 4.01(\mathrm{~d}, J=11.7 \mathrm{~Hz}, 1 \mathrm{H}), 4.50(\mathrm{~m}$, 1H), $5.68(\mathrm{~m}, 1 \mathrm{H}) \mathrm{ppm} ;{ }^{13} \mathrm{C}\left\{{ }^{1} \mathrm{H}\right\} \mathrm{NMR}\left(\mathrm{CDCl}_{3}, 125 \mathrm{MHz}\right) \delta-5.39,-5.29,14.4,18.3,19.3$, $23.1,24.5,26.0,26.1,29.8,30.2,30.9,32.4,35.3,40.9,66.6,71.7,127.8,140.3$ ppm; IR (neat) $3531,2919,2740,1659,1469,1254,1137 \mathrm{~cm}^{-1}$; HRMS calcd for $\mathrm{C}_{21} \mathrm{H}_{42} \mathrm{O}_{2} \mathrm{SiNa}$ $(\mathrm{M}+\mathrm{Na})^{+}: 377.2852$, found 377.2851 .

\section{6-Methyl-2-m-tolyl-heptan-2-ol (S23).}

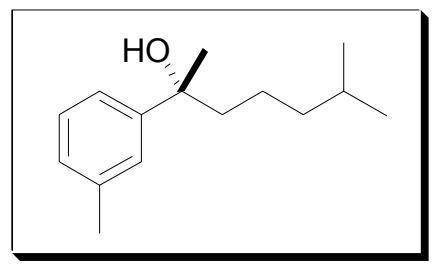

The crude product was purified by column chromatography on silica gel (hexanes / EtOAc : 96 / 4) to give the product as an oil: $[\alpha]^{20}{ }_{\mathrm{D}}=-4.8\left(\mathrm{c} 1.00, \mathrm{CHCl}_{3}\right) ;{ }^{1} \mathrm{H} \mathrm{NMR}\left(\mathrm{CDCl}_{3}, 500\right.$

$\mathrm{MHz}): \delta 0.75(\mathrm{~d}, J=6.6 \mathrm{~Hz}, 6 \mathrm{H}), 1.03-1.10(\mathrm{~m}, 3 \mathrm{H}), 1.17-1.22(\mathrm{~m}, 1 \mathrm{H}), 1.39-1.46(\mathrm{~m}$, $1 \mathrm{H}), 1.44(\mathrm{~s}, 3 \mathrm{H}), 1.63-1.74(\mathrm{~m}, 2 \mathrm{H}), 2.29(\mathrm{~s}, 3 \mathrm{H}), 6.97-6.98(\mathrm{~m}, 1 \mathrm{H}), 7.12-7.18(\mathrm{~m}, 3 \mathrm{H})$ ppm; ${ }^{13} \mathrm{C}\left\{{ }^{1} \mathrm{H}\right\}$ NMR $\left(\mathrm{CDCl}_{3}, 125 \mathrm{MHz}\right): \delta 22.0,22.1,22.9,23.0,28.2,30.6,39.7,44.8$, 
75.1, 122.2, 125.9, 127.6, 128.4, 138.0, 148.6 ppm; IR (neat) 3408, 2951, 2868, 1607, 1462, $1366 \mathrm{~cm}^{-1}$; HRMS calcd for $\mathrm{C}_{15} \mathrm{H}_{23}(\mathrm{M}-\mathrm{OH})^{+}: 203.1800$, found 203.1805.

6-(tert-Butyl-dimethyl-silanyloxy)-2-m-tolyl-hexan-2-ol (S24).

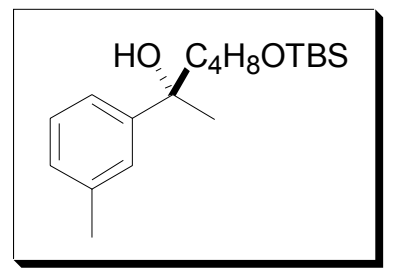

The crude product was purified by column chromatography on silica gel (hexanes / EtOAc : $97 / 3$ ) to give the product as an oil: $\left[\alpha{ }^{20}{ }_{\mathrm{D}}=-5.3\left(c\right.\right.$ 2.30, $\left.\mathrm{CHCl}_{3}\right) ;{ }^{1} \mathrm{H} \mathrm{NMR}\left(\mathrm{CDCl}_{3}, 500 \mathrm{MHz}\right)$ :

$\delta 0.04(\mathrm{~s}, 6 \mathrm{H}), 0.90(\mathrm{~s}, 9 \mathrm{H}), 1.48-1.50(\mathrm{~m}, 1 \mathrm{H}), 1.51-1.52(\mathrm{~m}, 1 \mathrm{H}), 1.52-1.54(\mathrm{~m}, 2 \mathrm{H})$, $1.56(\mathrm{~s}, 3 \mathrm{H}), 1.81-1.88(\mathrm{~m}, 2 \mathrm{H}), 2.39(\mathrm{~s}, 3 \mathrm{H}), 3.59(\mathrm{t}, J=6.5 \mathrm{~Hz}, 2 \mathrm{H}), 7.06-7.07(\mathrm{~m}, 1 \mathrm{H})$, 7.23-7.26 (m, 2H), 7.28 (s, $1 \mathrm{H}) \mathrm{ppm} ;{ }^{13} \mathrm{C}\left\{{ }^{1} \mathrm{H}\right\} \mathrm{NMR}\left(\mathrm{CDCl}_{3}, 125 \mathrm{MHz}\right): \delta-4.9,18.7$, $20.8,22.1,26.4,30.5,33.4,44.3,63.4,75.1,122.3,125.9,127.6,128.4,138.0,148.4$ ppm; IR (neat) 3413, 2930, 1607, 1462, $1255 \mathrm{~cm}^{-1}$; HRMS calcd for $\mathrm{C}_{19} \mathrm{H}_{33} \mathrm{OSi}$ (M$\mathrm{OH})^{+}: 305.2301$, found 305.2291.

7-Bromo-2-m-tolyl-heptan-2-ol (S25).

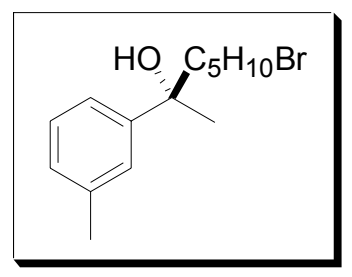

The crude product was purified by column chromatography on silica gel (hexanes / EtOAc : 92 / 8) to give the product as an oil: $[\alpha]_{\mathrm{D}}^{20}=-4.9\left(c\right.$ 0.87, $\left.\mathrm{CHCl}_{3}\right) ;{ }^{1} \mathrm{H} \mathrm{NMR}\left(\mathrm{CDCl}_{3}, 500 \mathrm{MHz}\right): \delta$

1.16-1.17 (m, 1H), 1.26-1.40 (m, 3H), $1.54(\mathrm{~s}, 3 \mathrm{H}), 1.76-1.83(\mathrm{~m}, 4 \mathrm{H}), 2.36(\mathrm{~s}, 3 \mathrm{H}), 3.34$ $(\mathrm{t}, J=6.8 \mathrm{~Hz}, 2 \mathrm{H}), 7.04-7.06(\mathrm{~m}, 1 \mathrm{H}), 7.18-7.25(\mathrm{~m}, 3 \mathrm{H}) \mathrm{ppm} ;{ }^{13} \mathrm{C}\left\{{ }^{1} \mathrm{H}\right\} \mathrm{NMR}\left(\mathrm{CDCl}_{3}\right.$, $125 \mathrm{MHz}): \delta 22.1,23.6,28.9,30.7,33.1,34.2,44.4,75.0,122.2,125.9,127.7,128.5$, 138.1, 148.3 ppm; IR (neat) 3437, 2937, 2859, $2361 \mathrm{~cm}^{-1}$; HRMS calcd for $\mathrm{C}_{14} \mathrm{H}_{20} \mathrm{Br}$ (M$\mathrm{OH})^{+}: 267.0749$, found 267.0752 .

6-Methyl-2-(3-trifluoromethyl-phenyl)-heptan-2-ol (S26). 


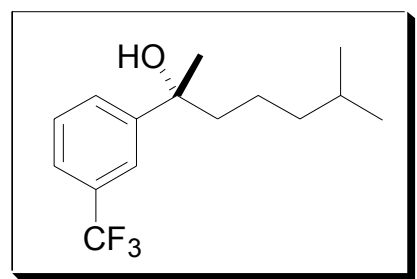

The crude product was purified by column chromatography on silica gel (hexanes / EtOAc : 94 / 6) to give the product as an oil: $[\alpha]{ }_{\mathrm{D}}^{20}=-4.0\left(\mathrm{c} 0.95, \mathrm{CHCl}_{3}\right) ;{ }^{1} \mathrm{H} \mathrm{NMR}\left(\mathrm{CDCl}_{3}, 500\right.$ MHz): $\delta 0.81(\mathrm{~d}, J=1.5 \mathrm{~Hz}, 3 \mathrm{H}), 0.82(\mathrm{~d}, J=1.5 \mathrm{~Hz}, 3 \mathrm{H})$, $1.10-1.15(\mathrm{~m}, 3 \mathrm{H}), 1.26-1.31(\mathrm{~m}, 1 \mathrm{H}), 1.47-1.50(\mathrm{~m}, 1 \mathrm{H}), 1.57(\mathrm{~s}, 3 \mathrm{H}), 1.76-1.83(\mathrm{~m}$, 2H), 7.43-7.46 (m, 1H), 7.49-7.51 (m, 1H), $7.61(\mathrm{~d}, J=7.5 \mathrm{~Hz}, 1 \mathrm{H}), 7.24(\mathrm{~s}, 1 \mathrm{H}) \mathrm{ppm}$; ${ }^{13} \mathrm{C}\left\{{ }^{1} \mathrm{H}\right\}$ NMR $\left(\mathrm{CDCl}_{3}, 125 \mathrm{MHz}\right): \delta 22.0,22.8,23.0,28.1,30.6,39.5,44.7,75.0,122.1$ $(\mathrm{d}, J=3.75 \mathrm{~Hz}), 123.7(\mathrm{~d}, J=3.75 \mathrm{~Hz}), 125.8,128.7,128.9,130.9(\mathrm{q}, J=31.2 \mathrm{~Hz})$, 149.6 ppm; IR (neat) 3390, 2954, 2872, 1460, 1325, $1166 \mathrm{~cm}^{-1}$; HRMS calcd for $\mathrm{C}_{15} \mathrm{H}_{19} \mathrm{~F}_{3}\left(\mathrm{M}-\mathrm{H}_{2} \mathrm{O}\right)^{+}:$256.1438, found 256.1444.

\section{3-(3-Chloro-phenyl)-7-methyl-octan-3-ol (S27).}

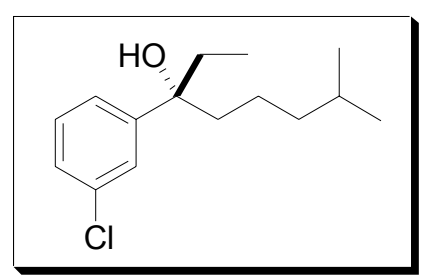

The crude product was purified by column chromatography on silica gel (hexanes / EtOAc : $96 / 4$ ) to give the product as an oil: $[\alpha]^{20}{ }_{\mathrm{D}}=+6.5\left(\mathrm{c} 1.50, \mathrm{CHCl}_{3}\right) ;{ }^{1} \mathrm{H} \mathrm{NMR}\left(\mathrm{CDCl}_{3}, 500\right.$ MHz): $\delta 0.75(\mathrm{t}, J=7.5 \mathrm{~Hz}, 3 \mathrm{H}), 0.80(\mathrm{~d}, J=6.6 \mathrm{~Hz}, 6 \mathrm{H})$, 1.01-1.07 (m, 1H), 1.09-1.15 (m, 2H), 1.24-1.32 (m, 1H), 1.44-1.49 (m. 1H), 1.69-1.86 (m, 4H), 7.19-7.27 (m, 3H), $7.40(\mathrm{~s}, 1 \mathrm{H}) \mathrm{ppm} ;{ }^{13} \mathrm{C}\left\{{ }^{1} \mathrm{H}\right\} \mathrm{NMR}\left(\mathrm{CDCl}_{3}, 125 \mathrm{MHz}\right): \delta 8.1$, $21.5,22.9,23.0,28.2,35.8,39.6,43.2,77.5,124.0,126.2,126.8,129.7,134.6,148.9$ ppm; IR (neat) 3460, 2943, 1595, $1467 \mathrm{~cm}^{-1}$; HRMS calcd for $\mathrm{C}_{15} \mathrm{H}_{22} \mathrm{Cl}(\mathrm{M}-\mathrm{OH})^{+}$: 237.1410, found 237.1406. 
7-(tert-Butyl-dimethyl-silanyloxy)-3-(3-chloro-phenyl)-heptan-3-ol (S28).

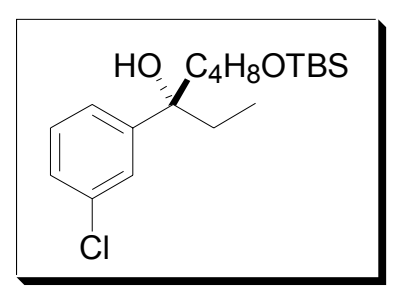

The crude product was purified by column chromatography on silica gel (hexanes / EtOAc : $95 / 5$ ) to give the product as an oil: $[\alpha]{ }_{\mathrm{D}}^{20}=+2.3\left(\right.$ c 2.20, $\left.\mathrm{CHCl}_{3}\right) ;{ }^{1} \mathrm{H} \mathrm{NMR}\left(\mathrm{CDCl}_{3}, 500\right.$ MHz): $\delta 0.038(\mathrm{~s}, 3 \mathrm{H}), 0.039(\mathrm{~s}, 3 \mathrm{H}), 0.79(\mathrm{t}, J=7.5 \mathrm{~Hz}, 3 \mathrm{H})$, $0.89(\mathrm{~s}, 9 \mathrm{H}), 1.13-1.14(\mathrm{~m}, 1 \mathrm{H}), 1.34-1.37(\mathrm{~m}, 1 \mathrm{H}), 1.48-1.52(\mathrm{~m}, 2 \mathrm{H}), 1.80-1.90(\mathrm{~m}$, 4H), $3.58(\mathrm{t}, J=6.5 \mathrm{~Hz}, 2 \mathrm{H}), 7.21-7.43(\mathrm{~m}, 3 \mathrm{H}), 7.43(\mathrm{~s}, 1 \mathrm{H}) \mathrm{ppm} ;{ }^{13} \mathrm{C}\left\{{ }^{1} \mathrm{H}\right\} \mathrm{NMR}$ $\left(\mathrm{CDCl}_{3}, 125 \mathrm{MHz}\right): \delta-4.9,8.1,18.7,20.3,26.3,33.3,35.8,42.6,63.3,77.4,124.0,126.2$ 126.8. 129.6, 134.6, 148.8 ppm; IR (neat) 3466, 2942, 2860, 2360, $1463 \mathrm{~cm}^{-1}$; HRMS calcd for $\mathrm{C}_{19} \mathrm{H}_{33} \mathrm{ClO}_{2} \mathrm{SiNa}(\mathrm{M}+\mathrm{Na})^{+}: 379.1836$, found 379.1835 .

6-Methyl-2-naphthalen-2-yl-heptan-2-ol (S29).

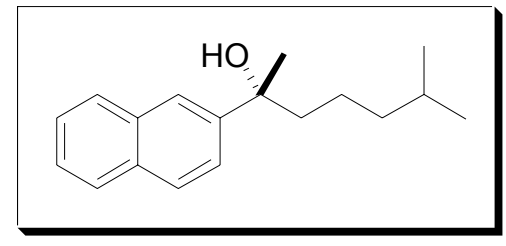

The crude product was purified by column chromatography on silica gel (hexanes / EtOAc : 95 / 5) to give the product as white solid: m.p. $86-88{ }^{\circ} \mathrm{C} ;[\alpha]^{20}{ }_{D}$

$=-1.3\left(c 1.90, \mathrm{CHCl}_{3}\right) ;{ }^{1} \mathrm{H} \mathrm{NMR}\left(\mathrm{CDCl}_{3}, 500 \mathrm{MHz}\right): \delta 0.81(\mathrm{~d}, J=6.6 \mathrm{~Hz}, 6 \mathrm{H}), 1.12-$ $1.21(\mathrm{~m}, 3 \mathrm{H}), 1.28-1.36(\mathrm{~m}, 1 \mathrm{H}), 1.45-1.59(\mathrm{~m}, 1 \mathrm{H}), 1.65(\mathrm{~s}, 3 \mathrm{H}) 1.82-1.95(\mathrm{~m}, 2 \mathrm{H})$, 7.45-7.50 (m, 2H), $7.54(\mathrm{dd}, J=8.5,1.65 \mathrm{~Hz}, 1 \mathrm{H}), 7.82-7.86(\mathrm{~m}, 3 \mathrm{H}), 7.92(\mathrm{~s}, 1 \mathrm{H}) \mathrm{ppm}$; ${ }^{13} \mathrm{C}\left\{{ }^{1} \mathrm{H}\right\}$ NMR $\left(\mathrm{CDCl}_{3}, 125 \mathrm{MHz}\right): \delta 22.2,22.9,23.0,28.2,30.7,39.7,44.6,75.4,123.5$, 124.1, 126.0, 126.4, 127.9, 128.2, 128.6, 132.6, 133.6, 145.9; IR (neat) 3421, 3056, 2951, 2866, 1600, $1462 \mathrm{~cm}^{-1}$; HRMS calcd for $\mathrm{C}_{18} \mathrm{H}_{22}\left(\mathrm{M}-\mathrm{H}_{2} \mathrm{O}\right)^{+}: 238.1722$, found 238.1728. 6-(tert-Butyl-dimethyl-silanyloxy)-2-naphthalen-2-yl-hexan-2-ol (S30). 


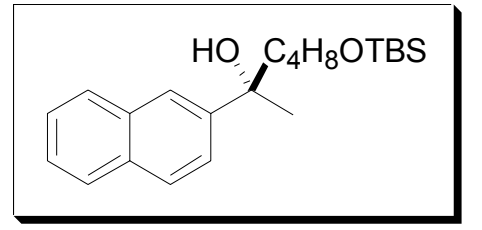

The crude product was purified by column chromatography on silica gel (hexanes / EtOAc : 93 / 7) to give the product as an oil: $[\alpha]_{\mathrm{D}}^{20}=-3.7\left(c\right.$ 2.30, $\left.\mathrm{CHCl}_{3}\right)$;

${ }^{1} \mathrm{H}$ NMR $\left(\mathrm{CDCl}_{3}, 500 \mathrm{MHz}\right): \delta 0.013$ (s, 3H), 0.017 (s, 3H), 0.86 (s, 9H), 1.22-1.24 (m, $1 \mathrm{H}), 1.35-1.38(\mathrm{~m}, 1 \mathrm{H}), 1.48-1.52(\mathrm{~m}, 2 \mathrm{H}), 1.66(\mathrm{~s}, 3 \mathrm{H}), 1.90-1.97(\mathrm{~m}, 2 \mathrm{H}), 3.57(\mathrm{t}, J=$ 6.5 Hz, 2H), 7.55-7.45 (m, 3H), 7.82-7.86 (m, 3H), $7.93(\mathrm{~s}, 1 \mathrm{H}) \mathrm{ppm} ;{ }^{13} \mathrm{C}\left\{{ }^{1} \mathrm{H}\right\} \mathrm{NMR}$ $\left(\mathrm{CDCl}_{3}, 125 \mathrm{MHz}\right): \delta-4.9,18.7,20.9,26.4,30.6,33.4,44.1,63.4,75.3,123.6,124.1$, 126.0, 126.4, 127.9, 128.2, 128.6, 132.7, 133.6, 145.8 ppm; IR (neat) 3421, 3056, 2928, 2856, $1461 \mathrm{~cm}^{-1}$; HRMS calcd for $\mathrm{C}_{22} \mathrm{H}_{34} \mathrm{O}_{2} \mathrm{SiNa}(\mathrm{M}+\mathrm{Na})^{+}: 381.2226$, found 381.2233.

7-Bromo-2-naphthalen-2-yl-heptan-2-ol (S31).

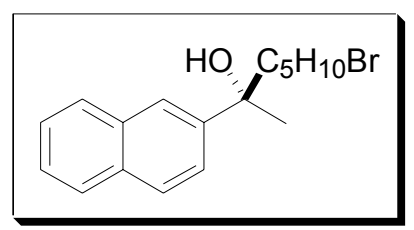

The crude product was purified by column chromatography on silica gel (hexanes / EtOAc : 92 / 8) to give the product as an oil: $[\alpha]{ }_{\mathrm{D}}^{20}=-1.6\left(c\right.$ 1.00, $\left.\mathrm{CHCl}_{3}\right) ;{ }^{1} \mathrm{H}$ NMR $\left(\mathrm{CDCl}_{3}, 500\right.$

$\mathrm{MHz}): \delta 1.16-1.17(\mathrm{~m}, 1 \mathrm{H}), 1.27-1.42(\mathrm{~m}, 3 \mathrm{H}), 1.66(\mathrm{~s}, 3 \mathrm{H}), 1.76-1.94(\mathrm{~m}, 4 \mathrm{H}), 3.33(\mathrm{t}, J$ $=6.5 \mathrm{~Hz}, 2 \mathrm{H}), 7.45-7.53(\mathrm{~m}, 3 \mathrm{H}), 7.82-7.86(\mathrm{~m}, 3 \mathrm{H}), 7.90(\mathrm{~s}, 1 \mathrm{H}) \mathrm{ppm} ;{ }^{13} \mathrm{C}\left\{{ }^{1} \mathrm{H}\right\} \mathrm{NMR}$ $\left(\mathrm{CDCl}_{3}, 125 \mathrm{MHz}\right): \delta 23.7,28.9,30.8,33.1,34.2,44.2,75.2,123.5,124.0,126.1,126.5$, 127.9, 128.3, 128.5, 132.7, 133.6, 145.6 ppm; IR (neat) 3422, 2936, $2857 \mathrm{~cm}^{-1}$; HRMS calcd for $\mathrm{C}_{17} \mathrm{H}_{21} \mathrm{BrO} \mathrm{M}$ : 320.0776 , found 320.0789 .

\section{3, 7-Dimethyl-1-phenyl-oct-1-en-3-ol (S32).}

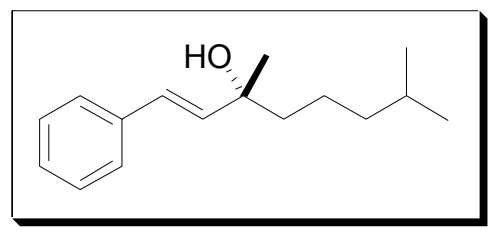

The crude product was purified by column chromatography on silica gel (hexanes / EtOAc : 95 / 5) to give the product as white solid: m.p. $70-71{ }^{\circ} \mathrm{C} ;[\alpha]^{20}{ }_{\mathrm{D}}$ $=-17.2\left(c\right.$ 2.50, $\left.\mathrm{CHCl}_{3}\right) ;{ }^{1} \mathrm{H} \mathrm{NMR}\left(\mathrm{CDCl}_{3}, 500 \mathrm{MHz}\right): \delta 0.80(\mathrm{~d}, J=6.6 \mathrm{~Hz}, 6 \mathrm{H}), 1.11-$ 
$1.14(\mathrm{~m}, 2 \mathrm{H}), 1.28-1.30(\mathrm{~m}, 1 \mathrm{H}), 1.31(\mathrm{~s}, 3 \mathrm{H}), 1.51-1.55(\mathrm{~m}, 4 \mathrm{H}), 6.21(\mathrm{~d}, J=16 \mathrm{~Hz}$ 1H), $6.51(\mathrm{~d}, J=16 \mathrm{~Hz}, 1 \mathrm{H}), 7.14-7.18(\mathrm{~m}, 1 \mathrm{H}), 7.24(\mathrm{t}, J=7.5 \mathrm{~Hz}, 2 \mathrm{H}), 7.31(\mathrm{~d}, J=7.0$ $\mathrm{Hz}, 2 \mathrm{H}) \mathrm{ppm} ;{ }^{13} \mathrm{C}\left\{{ }^{1} \mathrm{H}\right\} \mathrm{NMR}\left(\mathrm{CDCl}_{3}, 125 \mathrm{MHz}\right): \delta 22.2,23.03,23.06,28.3,28.6,39.8$, 43.6, 73.7, 126.8, 127.4, 127.8, 129.0, 137.3, 137.5 ppm; IR (neat) 3279, 2933, 1947, $1462 \mathrm{~cm}^{-1}$; HRMS calcd for $\mathrm{C}_{16} \mathrm{H}_{24} \mathrm{O} \mathrm{M}^{+}$: 232.1827, found 232.1828 .

7-(tert-Butyl-dimethyl-silanyloxy)-3-methyl-1-phenyl-hept-1-en-3-ol (S33).

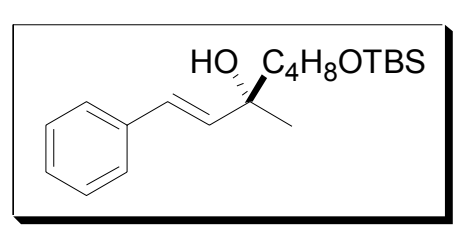

The crude product was purified by column chromatography on silica gel (hexanes / EtOAc : $92 / 8$ ) to give the product as an oil: $[\alpha]^{20}{ }_{\mathrm{D}}=-12.7$ (c 2.25, $\left.\mathrm{CHCl}_{3}\right) ;{ }^{1} \mathrm{H} \mathrm{NMR}\left(\mathrm{CDCl}_{3}\right.$,

$500 \mathrm{MHz}): \delta-0.07(\mathrm{~s}, 3 \mathrm{H}),-0.04(\mathrm{~s}, 3 \mathrm{H}), 0.80(\mathrm{~s}, 9 \mathrm{H}), 1.31(\mathrm{~s}, 3 \mathrm{H}), 1.33-1.37(\mathrm{~m}, 2 \mathrm{H})$, $1.45-1.48(\mathrm{~m}, 2 \mathrm{H}), 1.54-1.59(\mathrm{~m}, 2 \mathrm{H}), 3.54(\mathrm{t}, J=6.5 \mathrm{~Hz}, 2 \mathrm{H}), 6.20(\mathrm{~d}, J=16 \mathrm{~Hz}, 1 \mathrm{H})$, $6.51(\mathrm{~d}, J=16 \mathrm{~Hz}, 1 \mathrm{H}), 7.13-7.15(\mathrm{~m}, 1 \mathrm{H}), 7.22-7.25(\mathrm{~m}, 2 \mathrm{H}), 7.29-7.31(\mathrm{~m}, 2 \mathrm{H}) \mathrm{ppm}$; ${ }^{13} \mathrm{C}\left\{{ }^{1} \mathrm{H}\right\}$ NMR $\left(\mathrm{CDCl}_{3}, 125 \mathrm{MHz}\right): \delta-4.8,18.8,20.8,26.4,28.6,33.5,43.0,63.4,73.7$, $126.8,127.5,127.7,128.9,137.2,137.5 \mathrm{ppm}$; IR (neat) 3389, 2929, 2856, $1471 \mathrm{~cm}^{-1}$; HRMS calcd for $\mathrm{C}_{20} \mathrm{H}_{33} \mathrm{OSi}(\mathrm{M}-\mathrm{OH})^{+}: 317.2301$, found 317.2299.

\section{8-Bromo-3-methyl-1-phenyl-oct-1-en-3-ol (S34).}

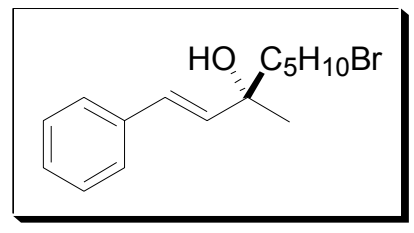

The crude product was purified by column chromatography on silica gel (hexanes / EtOAc : $95 / 5$ ) to give the product as an oil: $[\alpha]_{\mathrm{D}}^{20}=-14.9\left(\mathrm{c} 1.30, \mathrm{CHCl}_{3}\right) ;{ }^{1} \mathrm{H} \mathrm{NMR}\left(\mathrm{CDCl}_{3}, 500\right.$

MHz): $\delta 1.38(\mathrm{~s}, 3 \mathrm{H}), 1.35-1.48(\mathrm{~m}, 4 \mathrm{H}), 1.58-1.66(\mathrm{~m}, 2 \mathrm{H}), 1.83-1.88(\mathrm{~m}, 2 \mathrm{H}), 3.39(\mathrm{t}, J$ $=6.8 \mathrm{~Hz}, 2 \mathrm{H}), 6.26(\mathrm{~d}, J=16 \mathrm{~Hz}, 1 \mathrm{H}), 6.58(\mathrm{~d}, J=16 \mathrm{~Hz}, 1 \mathrm{H}), 7.21-7.25(\mathrm{~m}, 1 \mathrm{H}), 7.29-$ $7.32(\mathrm{~m}, 2 \mathrm{H}), 7.37-7.38(\mathrm{~m}, 2 \mathrm{H}) \mathrm{ppm} ;{ }^{13} \mathrm{C}\left\{{ }^{1} \mathrm{H}\right\} \mathrm{NMR}\left(\mathrm{CDCl}_{3}, 125 \mathrm{MHz}\right): \delta 23.7,28.8$, 
29.0, 33.2, 34.2, 43.1, 73.6, 126.8, 127.6, 127.8, 129.0, 137.0, 137.4 ppm; IR (neat) 3402, 2935, 2857, $1448 \mathrm{~cm}^{-1}$; HRMS calcd for $\mathrm{C}_{15} \mathrm{H}_{20} \mathrm{Br}(\mathrm{M}-\mathrm{OH})^{+}:$279.0749, found 279.0747.

\section{Conditions for the Determination of Enantiomeric Excesses.}

The racemic alcohols were prepared by addition of Grignard reagents or in situ generated organozinc reagents with racemic catalysts to the corresponding ketones, and then analyzed by chiral capillary GC or HPLC for ee determination. The specifications for the GC analyses were as follows: Fused silica chiral capillary column (Supelco $\beta$-Dex 120): $30 \mathrm{~m} \mathrm{x} 0.25 \mathrm{~mm}$ (id) $\times 0.25 \mu \mathrm{m}$ film thickness. Carrier gas: nitrogen. Inlet temperature: $250{ }^{\circ} \mathrm{C}$. Detector: FID, $270{ }^{\circ} \mathrm{C}$. The conditions for the resolution of the racemates by $\mathrm{GC}$ are given below.

2-(tert-Butyl-dimethyl-silanyloxymethyl)-1-methyl-cyclohex-2-enol (S1). $\mathrm{t}_{1}=170.5$ $\min , \mathrm{t}_{2}=189.0 \mathrm{~min}\left(100{ }^{\circ} \mathrm{C}\right.$ for $170 \mathrm{~min}, \operatorname{ramp} 1{ }^{\circ} \mathrm{C} / \mathrm{min}$, and $110{ }^{\circ} \mathrm{C}$ for $\left.60 \mathrm{~min}\right)$. 2-(tert-Butyl-dimethyl-silanyloxymethyl)-1-ethyl-cyclohex-2-enol (S2). $\mathrm{t}_{1}=221.3 \mathrm{~min}$, $\mathrm{t}_{2}=224.5 \mathrm{~min}\left(100{ }^{\circ} \mathrm{C}\right.$ for $170 \mathrm{~min}$, ramp $1{ }^{\circ} \mathrm{C} / \mathrm{min}$, and $110{ }^{\circ} \mathrm{C}$ for $\left.60 \mathrm{~min}\right)$.

4-Methyl-4,5,6,7-tetrahydro-benzofuran-4-ol (S3). $\mathrm{t}_{1}=98.4 \mathrm{~min}, \mathrm{t}_{2}=100.4 \min (100$ $\left.{ }^{\circ} \mathrm{C}, 0.7 \mathrm{~mL} / \mathrm{min}\right)$.

4-Ethyl-4,5,6,7-tetrahydro-benzofuran-4-ol (S4). $\mathrm{t}_{1}=174.0 \mathrm{~min}, \mathrm{t}_{2}=175.4 \mathrm{~min}(100$ $\left.{ }^{\circ} \mathrm{C}, 0.7 \mathrm{~mL} / \mathrm{min}\right)$.

2,4,4-Trimethyl-1-octyl-cyclohex-2-enol (S19). $t_{1}=97.6 \mathrm{~min}, \mathrm{t}_{2}=107.2 \min \left(130{ }^{\circ} \mathrm{C}\right.$, $1.4 \mathrm{~mL} / \mathrm{min})$. 
1-(4-chloro-butyl)-2,4,4-trimethyl-cyclohex-2-enol (S20). $t_{1}=32.3 \mathrm{~min}, \mathrm{t}_{2}=34.7 \mathrm{~min}$ $\left(145^{\circ} \mathrm{C}, 1.5 \mathrm{~mL} / \mathrm{min}\right)$.

2,2-Dimethyl-propionic acid 4-(1-hydroxy-2,4,4-trimethyl-cyclohex-2-enyl)-butyl ester (S21). $\mathrm{t}_{1}=48.0 \mathrm{~min}, \mathrm{t}_{2}=50.6 \mathrm{~min}\left(160^{\circ} \mathrm{C}, 1.6 \mathrm{~mL} / \mathrm{min}\right)$.

2-(tert-Butyl-dimethyl-silanyloxymethyl)-1-octyl-cyclohex-2-enol (S22). $\mathrm{t}_{1}=187.0$ $\min , \mathrm{t}_{2}=190.0 \min \left(150^{\circ} \mathrm{C}, 1.5 \mathrm{~mL} / \mathrm{min}\right)$.

6-Methyl-2-m-tolyl-heptan-2-ol (S23). $\mathrm{t}_{1}=188.9 \mathrm{~min}, \mathrm{t}_{2}=195.8 \mathrm{~min}\left(115^{\circ} \mathrm{C}, 1.0\right.$ $\mathrm{mL} / \mathrm{min})$.

3-(3-Chloro-phenyl)-7-methyl-octan-3-ol (S27). $\mathrm{t}_{1}=255.7 \mathrm{~min}, \mathrm{t}_{2}=263.7 \mathrm{~min}\left(125^{\circ} \mathrm{C}\right.$, $1.0 \mathrm{~mL} / \mathrm{min})$.

Chiral HPLC analyses were performed using a Chiralcel OD-H column unless otherwise specified.

5-Methyl-8,9-dihydro-5 $\boldsymbol{H}$-benzocyclohepten-5-ol (S5). $\mathrm{t}_{1}=22.7 \mathrm{~min}, \mathrm{t}_{2}=24.9 \mathrm{~min}$ (hexanes / 2-propanol : $98 / 2,0.5 \mathrm{~mL} / \mathrm{min}$ ).

5-Ethyl-8,9-dihydro-5H-benzocyclohepten-5-ol (S6). $\mathrm{t}_{1}=14.6 \mathrm{~min}, \mathrm{t}_{2}=16.4 \mathrm{~min}$ (hexanes / 2-propanol : $98 / 2,0.5 \mathrm{~mL} / \mathrm{min}$ ).

2-m-Tolyl-decan-2-ol (S7). $\mathfrak{t}_{1}=16.8 \mathrm{~min}, \mathrm{t}_{2}=18.3 \mathrm{~min}$ (hexanes / 2-propanol : $97 / 3$, $0.3 \mathrm{~mL} / \mathrm{min})$.

6-Chloro-2-m-tolyl-hexan-2-ol (S8). $\mathrm{t}_{1}=90.4 \mathrm{~min}, \mathrm{t}_{2}=105.2 \mathrm{~min}$ (hexanes $/ 2$ propanol : 99.4 / 0.6, $0.3 \mathrm{~mL} / \mathrm{min})$. 
2, 2-Dimethyl-propionic acid 5-hydroxy-5-m-tolyl-hexyl-ester (S9). $\mathrm{t}_{1}=50.8 \mathrm{~min}, \mathrm{t}_{2}=$ $52.9 \mathrm{~min}$ (hexanes / 2-propanol : 99.2 / 0.8, $0.5 \mathrm{~mL} / \mathrm{min}$ ).

2-Naphthalen-2-yl-decan-2-ol (S10). $\mathrm{t}_{1}=17.6 \mathrm{~min}, \mathrm{t}_{2}=23.4 \mathrm{~min}$ (hexanes $/ 2$-propanol : $95 / 5,0.5 \mathrm{~mL} / \mathrm{min})$.

6-Chloro-2-naphthalen-2-yl-hexan-2-ol (S11). $\mathrm{t}_{1}=42.0 \mathrm{~min}, \mathrm{t}_{2}=52.4 \mathrm{~min}$ (hexanes $/ 2$ propanol : 95 / 5, $0.5 \mathrm{~mL} / \mathrm{min})$.

2, 2-Dimethyl-propionic acid 5-hydroxy-5-(3-trifluoromethyl-phenyl)-hexyl-ester (S12). $\mathrm{t}_{1}=20.3 \mathrm{~min}, \mathrm{t}_{2}=23.3 \mathrm{~min}$ (hexanes $/ 2$-propanol : $98 / 2,0.5 \mathrm{~mL} / \mathrm{min}$ ).

5-Octyl-8,9-dihydro-5H-benzocyclohepten-5-ol (S13). $\mathrm{t}_{1}=13.0 \mathrm{~min}, \mathrm{t}_{2}=15.9 \mathrm{~min}$ (hexanes / 2-propanol : $98 / 2,0.5 \mathrm{~mL} / \mathrm{min}$ ).

5-(4-Chloro-butyl)-8,9-dihydro-5 $\boldsymbol{H}$-benzocyclohepten-5-ol $(\mathbf{S 1 4}) . \mathrm{t}_{1}=14.4 \mathrm{~min}, \mathrm{t}_{2}=$ $28.9 \mathrm{~min}$ (hexanes / 2-propanol : 95 / 5, $0.5 \mathrm{~mL} / \mathrm{min}$ ).

2,2-Dimethyl-propionic acid 4-(5-hydroxy-8,9-dihydro-5H-benzocyclohepten-5-yl)butyl ester $(\mathbf{S 1 5}) . \mathrm{t}_{1}=13.2 \mathrm{~min}, \mathrm{t}_{2}=17.4 \mathrm{~min}$ (hexanes $/ 2$-propanol : $\left.95 / 5,0.5 \mathrm{~mL} / \mathrm{min}\right)$. 4-Octyl-4,5,6,7-tetrahydro-benzofuran-4-ol (S16). $\mathrm{t}_{1}=9.8 \mathrm{~min}, \mathrm{t}_{2}=11.4 \mathrm{~min}$ (hexanes / 2-propanol : 95 / 5, $0.5 \mathrm{~mL} / \mathrm{min})$.

4-(4-Chloro-butyl)-4,5,6,7-tetrahydro-benzofuran-4-ol (S17). $\mathrm{t}_{1}=16.2 \mathrm{~min}, \mathrm{t}_{2}=18.9$ $\min$ (hexanes / 2-propanol : 95 / 5, $0.5 \mathrm{~mL} / \mathrm{min}$ ).

2,2-Dimethyl-propionic acid 4-(4-hydroxy-4,5,6,7-tetrahydro-benzofuran-4-yl)-butyl ester (S18). $\mathrm{t}_{1}=14.4 \mathrm{~min}, \mathrm{t}_{2}=16.2 \mathrm{~min}$ (hexanes / 2-propanol : $95 / 5,0.5 \mathrm{~mL} / \mathrm{min}$ ). 6-(tert-Butyl-dimethyl-silanyloxy)-2-m-tolyl-hexan-2-ol (S24). $\mathrm{t}_{1}=42.0 \mathrm{~min}, \mathrm{t}_{2}=45.5$ min (hexanes / 2-propanol : 97.5/2.5, $0.5 \mathrm{~mL} / \mathrm{min}$ ). 
7-Bromo-2-m-tolyl-heptan-2-ol (S25). $\mathrm{t}_{1}=17.4 \mathrm{~min}, \mathrm{t}_{2}=20.3 \mathrm{~min}$ (hexanes / 2-propanol : 97 / 3, $0.5 \mathrm{~mL} / \mathrm{min})$.

6-Methyl-2-(3-trifluoromethyl-phenyl)-heptan-2-ol (S26). $t_{1}=14.3 \mathrm{~min}, \mathrm{t}_{2}=19.3 \mathrm{~min}$ (hexanes / 2-propanol : 99.6 / 0.4, $0.5 \mathrm{~mL} / \mathrm{min}$, chiral AS column).

7-(tert-Butyl-dimethyl-silanyloxy)-3-(3-chloro-phenyl)-heptan-3-ol (S28). $\quad t_{1}=11.8$ $\min , \mathrm{t}_{2}=12.7 \mathrm{~min}$ (hexanes / 2-propanol : 98.4/ 1.6, 0.5 $\mathrm{mL} / \mathrm{min}$ ).

6-Methyl-2-naphthalen-2-yl-heptan-2-ol (S29). $\mathrm{t}_{1}=24.4 \mathrm{~min}, \mathrm{t}_{2}=33.3 \mathrm{~min}$ (hexanes / 2-propanol : 97 / 3, $0.5 \mathrm{~mL} / \mathrm{min})$.

6-(tert-Butyl-dimethyl-silanyloxy)-2-naphthalen-2-yl-hexan-2-ol (S30). $\mathrm{t}_{1}=38.0 \mathrm{~min}$, $\mathrm{t}_{2}=41.5 \mathrm{~min}$ (hexanes / 2-propanol : $\left.98 / 2,0.5 \mathrm{~mL} / \mathrm{min}\right)$.

7-Bromo-2-naphthalen-2-yl-heptan-2-ol (S31). $\mathrm{t}_{1}=17.0 \mathrm{~min}, \mathrm{t}_{2}=26.2 \mathrm{~min}$ (hexanes / 2-propanol : 95 / 5, $1.0 \mathrm{~mL} / \mathrm{min})$.

3, 7-Dimethyl-1-phenyl-oct-1-en-3-ol (S32). $\mathrm{t}_{1}=23.5 \mathrm{~min}, \mathrm{t}_{2}=26.0 \mathrm{~min}$ (hexanes $/ 2$ propanol : 97 / 3, $0.5 \mathrm{~mL} / \mathrm{min})$.

7-(tert-Butyl-dimethyl-silanyloxy)-3-methyl-1-phenyl-hept-1-en-3-ol (S33). $t_{1}=21.6$ $\min , \mathrm{t}_{2}=29.4 \min$ (hexanes / 2-propanol : $97 / 3,0.5 \mathrm{~mL} / \mathrm{min}$ ).

8-Bromo-3-methyl-1-phenyl-oct-1-en-3-ol (S34). $\mathrm{t}_{1}=23.3 \mathrm{~min}, \mathrm{t}_{2}=25.3 \mathrm{~min}$ (hexanes / 2-propanol : 90 / 10, $0.4 \mathrm{~mL} / \mathrm{min})$.

\section{Determination of Absolute Stereochemistry}

$(S)$ - and (R)-2-Phenyl-2-butanol (entry 1 and 17 in Table 1) already determined in previous paper $^{3}$

(R)-3-Phenyl-heptan-3-ol (entry 9 in Table 1) $[\alpha]^{20}{ }_{\mathrm{D}}=-1.1(c 2.4, \mathrm{MeOH})$ 
$\left[\text { Published }[\alpha]_{\mathrm{D}}^{20}=-1.8(c 2.4, \mathrm{MeOH}, 86 \% \text { ee })\right]^{4}$

(R)-2-Benzylidene-1-ethyl-cyclohexanol (entry 11 in Table 2) Determined with the product by one further transformation in eq. 1

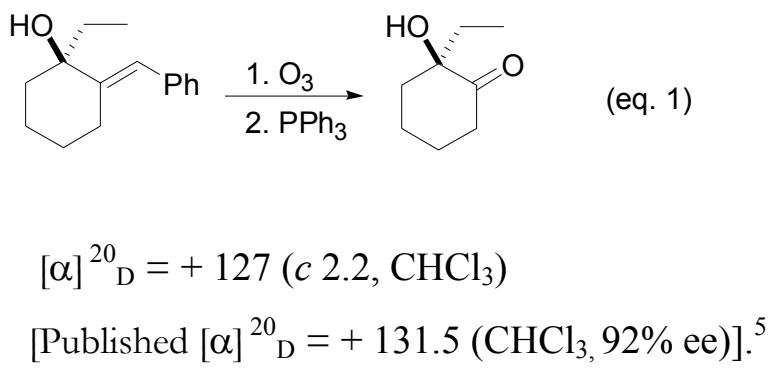

(R)-1,2-Dimethyl-cyclopent-2-enol (entry 14 in Table 2) Determined with the product by two further transformation in eq. 2

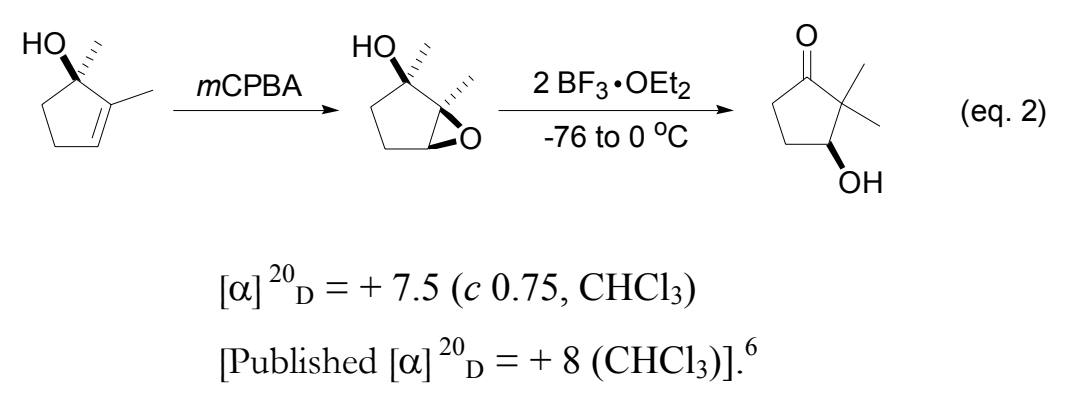

\section{References.}

1) Rozema, M. J.; Sidduri, A.; Knochel, P. J. Org. Chem, 1992, 57, 1956-1958.

2) Langer, F.; Schwink, L.; Devasagayaraj, A.; Chavant, P.-Y.; Knochel, P. J. Org. Chem, 1996, 61, 8229-8243.

3) Garcia, C.; LaRochelle, L. K.; Walsh, P. J. Am. Chem. Soc. 2002, 124, 10970-10971.

4) Ramon, D. J.; Yus, M. Tetrahedron Lett., 1998, 39, 1239-1242.

5) Fujisawa, T.; Watanabe, M.; Sato, T. Chem. Lett. 1984, 2055-2058.

6) Lee, S.-F.; Barth, G.; Djerassi, C. J. Am. Chem. Soc. 1981, 103, 295-301. 